\title{
Mid-Link Optical Phase Conjugation in Lumped Optical Transmission Systems
}

\author{
Mohammad A. Z. Al-Khateeb, Mary E. McCarthy, Christian Costa Sánchez, and Andrew D. Ellis \\ Aston Institute of Photonic Technologies (AIPT), Aston University, Birmingham, B4 7ET, UK \\ Authore-mail address: alkhamaz@aston.ac.uk
}

\begin{abstract}
We report an analytical description of four wave mixing in lumped optical transmission systems deploying mid-link optical phase conjugator, and provide corroborating simulation results matching theoretical predictions for four wave mixing and for modulated signals. OCIS codes: (190.4380) Nonlinear optics, four-wave mixing; (060.4510) Optical communications.
\end{abstract}

\section{Introduction}

The performance of optical communication systems is optimized by calibrating optical signal power trading-off between linear and nonlinear noise generated within the system; which is described in the literature as nonlinear Shannon limit [1]. Four wave mixing (FWM) is a dominant nonlinear effect [2]; which scales cubically with signal power in optical fiber transmission systems with efficient spectral shaping (e.g. orthogonal frequency division multiplexing (OFDM) and Nyquist spaced channel). FWM have been theoretically described [3] for lumped optical transmission systems where it was used later to approximate system nonlinear performance [2]. Mid-link optical phase conjugation (OPC) [4] is an all optical technique that can lead to significant compensation of inter- and intrachannel nonlinear mixing facilitating a higher system performance. Spectral inversion in the OPC means that the deterministic nonlinear signal-signal interactions produced by FWM (that have accumulated in the first half of the link) can be unwound by symmetrical propagation in the second half of the link. The efficiency of the nonlinearity compensation in mid-link OPC system is directly dependent on the power profile and dispersion symmetry in reference to the mid-link point. This can be achieved using ideal Raman (quasi lossless) amplified system [5] leads to full compensation signal-signal nonlinear compensation which reveals the nondeterministic signal-signal interaction due to polarization mode dispersion (PMD) [6] and signal-noise interaction [7,8].

In this paper, we present and numerically validate, for the first time, an analytical closed form description of FWM in lumped optical transmission system that deploys mid-link OPC. In Section 3, we will validate in simulation that the strongly phase matched signals show significant minimization FWM products power due to the mid-link OPC as predicted by the analytical description. In section 4, we will use the analytical findings of FWM in lumped optical transmission system (with or without mid-link OPC) to calculate the nonlinear thresholds for different cases of span length and signal bandwidth of $112 \mathrm{Gbps}$ PM-QPSK within $0.1 \mathrm{~dB}$ margin of error.

\section{Theory}

The power of FWM products generated in a system is largely dependent on the signals power profile and whether phase conjugation appear along the link or not. In lumped optical amplified system (without OPC) the FWM products power generated from the mixing of frequency tones $\left(f_{i}, f_{j}\right.$, and $\left.f_{k}\right)$ can be described in a closed form [3] as:

$$
P_{F W M}\left(f_{i}, f_{j}, f_{k}\right)=P_{i} P_{j} P_{k}\left(\frac{\mathrm{D} \gamma}{3}\right)^{2} \frac{\sin (\mathrm{N} \Delta \beta \mathrm{L} / 2)^{2}}{\sin (\Delta \beta \mathrm{L} / 2)^{2}}\left[\frac{\left(1-\mathrm{e}^{-\alpha \mathrm{L}}\right)^{2}+4 \mathrm{e}^{-\alpha \mathrm{L}} \sin (\Delta \beta \mathrm{L} / 2)^{2}}{\alpha^{2}+\Delta \beta^{2}}\right]
$$

By the same derivation technique, FWM power in lumped optical amplified system deploying mid-link OPC and can be written in a closed form as follows:

$$
P_{\mathrm{FWM}}\left(f_{i}, f_{j}, f_{k}\right)=P_{i} P_{j} P_{k}\left\{\frac{2 \mathrm{D} \gamma}{3\left(\alpha^{2}+\Delta \beta^{2}\right)} \frac{\sin (\mathrm{N} \Delta \beta \mathrm{L} / 4)}{\sin (\Delta \beta \mathrm{L} / 2)}\left[\alpha\left(e^{-\alpha L}+1\right) \sin \left(\frac{\Delta \beta \mathrm{L}}{2}\right)+\Delta \beta\left(e^{-\alpha L}-1\right) \cos \left(\frac{\Delta \beta \mathrm{L}}{2}\right)\right]\right\}^{2}
$$

Where $D$ is the degeneracy factor, $\gamma$ and $\alpha$ are respectively the nonlinear and attenuation coefficients of the fiber, $L$ is span length, $N$ is the number of spans in the system, and phase matching coefficient $\Delta \beta$ between mixing tones due to fiber dispersion coefficient $\beta_{2}$ can be defined as: $\Delta \beta=-4 \pi^{2} \beta_{2}\left(f_{i}-f_{k}\right)\left(f_{j}-f_{k}\right)$. By observing Eqns. (1) and (2), it is clear that the FWM efficiency in the case of mid-link OPC system oscillates (as a function of phase matching) at half the frequency $(\sin (N \Delta \beta L / 4))$ of oscillation for the case where OPC is omitted $(\sin (N \Delta \beta L / 2))$. This can be explained by the discontinuity that the OPC causes in the FWM accumulation by splitting the link into two sections. 


\section{Simulation results: Four Wave Mixing}

Eqn. (1) and (2) are validated using a simulation model using VPITransmissionMaker 9.5 implementing a lumped optical system with or without an ideal OPC (conjugating optical field by MatlabR2014a module) placed at midlink. Fig. 1 shows the simulation setup, where we injected two CW lasers into the system and at the output of the link we measured the FWM products power as we changed the frequency separation $(\Delta f)$ between the two lasers; this measurement intended to check the effect of phase matching effect on FWM process. The laser power was $10 \mathrm{dBm}$, frequency separation between lasers was swept from $0.7 \mathrm{GHz}$ to $10 \mathrm{GHz}$, and fiber parameters are: length of $100 \mathrm{~km}$ with $0.2 \mathrm{~dB} / \mathrm{km}$ attenuation constant, dispersion of $16 \mathrm{ps} /(\mathrm{nm} . \mathrm{km})$, and nonlinear coefficient of $1.33(\mathrm{~W} . \mathrm{km})^{-1}$.

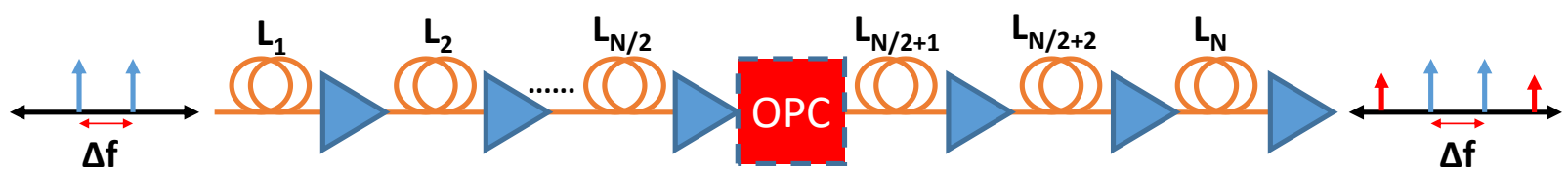

Fig. 1 FWM measurement simulation model.
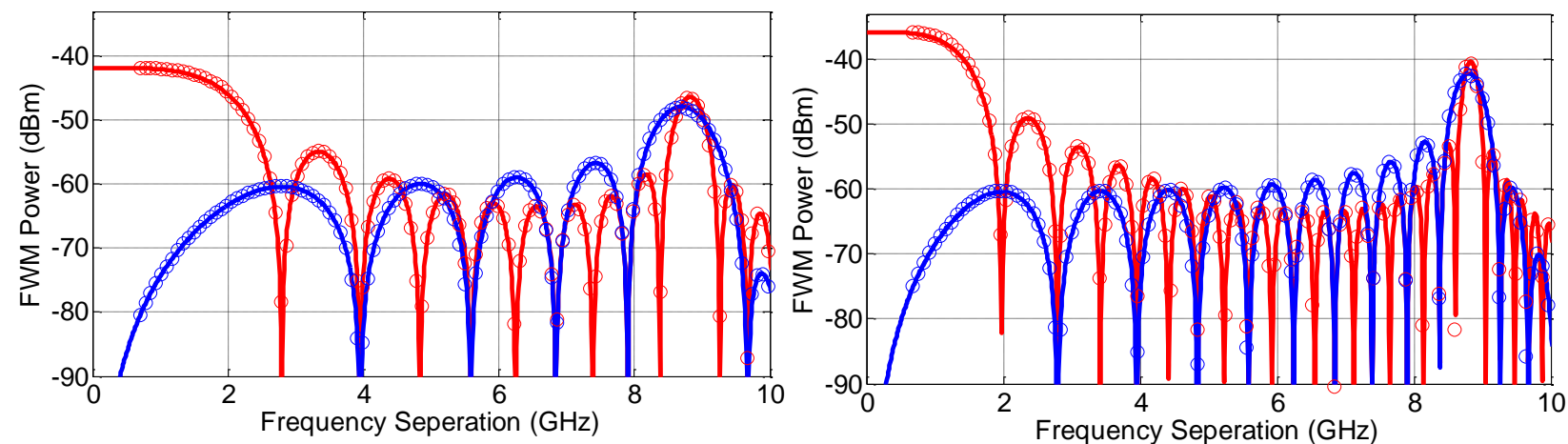

Fig. 2 FWM power as a function of frequency separation for (left) 10x100km and (right) 20x100km lumped systems. (Solid lines) theoretical prediction, (opened circles) simulation results, (red) without mid-link OPC, (blue) with mid-link OPC.

Fig. 2 shows FWM products power as function of frequency separation calculated from Eqns. (1) and (2) and the simulation results; the figure shows a good agreement between the simulation results and the theoretical predictions. From the figure, we can notice that FWM power resulted from the system that deploys mid-link OPC shows half the frequency of oscillation (as function $\Delta f$ ) compared to the case of a system without an OPC. When doubling the number of system spans (from 10 to 20) for the case of a system without mid-link OPC, the FWM components power quadruples (increases by $6 \mathrm{~dB}$ ) at strongly phase matched (low frequency separation between lasers) this is directly related to the fact that as $\Delta \beta \rightarrow 0$ in Eqn. (1) the resulted FWM power scales by $N^{2}$. On the other hand, for the case of a system with mid-link OPC we can see from Eqn. (2) that FWM power goes to zero as $\Delta \beta \rightarrow 0$ meaning that the mid-link OPC compensated the strongly phase matched nonlinear FWM product.

\section{Simulation results: Modulated optical signal performance}

In this section, we will use the analytical findings of FWM in lumped optical transmission system (with or without mid-link OPC) to calculate the nonlinear thresholds for different cases of span length and signal bandwidth. The nonlinear noise generation efficiency factor in optical transmission systems can be found by calculating a double integration of FWM (from Eqn. 1 and 2) over the signal bandwidth [2]. The signal to noise ratio (which equals to $Q^{2}$ factor of QPSK modulated signal) can be calculated by finding the ratio between signal launched power and the sum of: the linear ASE noise generated from amplifiers, the nonlinear signal-signal interaction noise, and the signal-noise interaction noise as in [8]. To verify Eqn. (2), we have used the model from previous section to transmit 112Gbps PM-QPSK modulated at $1550 \mathrm{~nm}$ carrying two pseudo random bit sequences (PRBS) on each polarization; this signal propagates in $20 x 100 \mathrm{~km}$ lumped system that uses erbium doped fiber amplifiers (EDFA's) with noise figure of $4 \mathrm{~dB}$. The transmitted signals were Nyquist pulse shaped with a roll off factor of 0.01 , the total number of transmitted bits was $2^{15}$ bits per polarization, and the modulated optical signals have been simulated with 16 samples per symbol. At the receiver side, signals were coherently detected with 8 samples per symbol and then passed to the DSP module, implemented in Matlab, to compensate for dispersion (in the case of system without mid-link OPC). The signals then were down-sampled to two samples per symbol; then passed to a phase recovery block which was performed using Viterbi-Viterbi algorithm with an averaging window of 21 . The $Q^{2}$ of received signals were calculated from the error vector magnitude (EVM). 

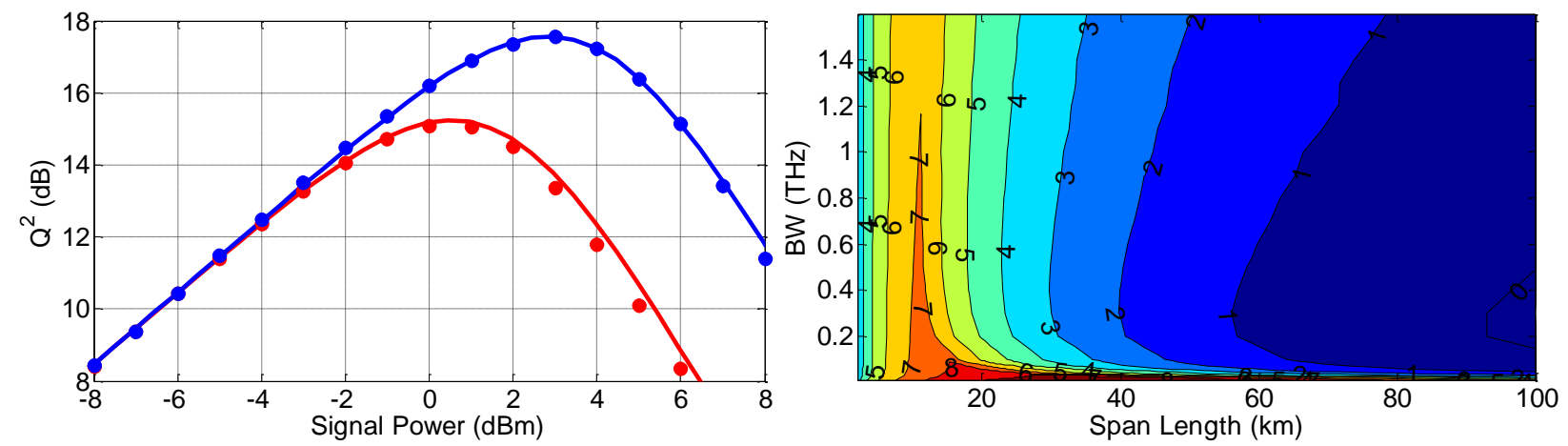

Fig. 3 (Left) received $Q^{2}$ of single channel 112Gbps PM-QPSK through 20x100km system as a function of launched optical signal power, (solid line) theory, (filled dots) simulation results, (red) without mid-link OPC, and (blue) with mid-link OPC. (right) theoretical evaluation of $Q^{2}$ improvement as a function of span length and signal bandwidth.

Fig. 3 (left) shows that single channel transmission in a system where a mid-link OPC is installed can improve the $Q^{2}$ factor by $2.3 \mathrm{~dB}$ due to significant compensation of strongly phase matched signal-signal FWM interaction. The simulation results show a good agreement (within $0.1 \mathrm{~dB}$ margin of error) with the theoretical prediction resulted from numerically integrated Eqns. (1) and (2). Fig.3 (right) shows the amount of $Q^{2}$ improvement that can be achieved in $2000 \mathrm{~km}$ link that deploys mid-link OPC as a function span length and signal bandwidth. As the system span length decreases, the mid-link OPC system starts to show an increasing $Q^{2}$ improvement ranging from 0 to $7.2 \mathrm{~dB}$ (for $1 \mathrm{THz}$ system) due to the compensation of signal-signal FWM interaction which can be fully eliminated as span length goes to zero resulting a quasi-lossless fiber $\left(\alpha \rightarrow 0\right.$ and $P_{F W M} \rightarrow 0$ as can be seen from Eqn. (2)). Due to the signal-noise interaction, the improvement level of $Q^{2}$ (for $1 \mathrm{THz}$ system) starts to be degraded as the span length become shorter than $10 \mathrm{~km}$. This degradation is due higher amount of ASE noise $\left(N I_{n}\right)$, and growth of the value of nonlinear scaling factor for signal-noise interaction. When system bandwidth increases for a given span length, we can notice that improvement in $Q^{2}$ factor degrades from $8.4 \mathrm{~dB}$ to $1.4 \mathrm{~dB}$ for $50 \mathrm{~km}$ span length system within the $200 \mathrm{GHz}$ bandwidth range, while the degradation of $Q^{2}$ degrades at slower pace from $8.9 \mathrm{~dB}$ to $5.2 \mathrm{~dB}$ for $20 \mathrm{~km}$ system within $1.5 \mathrm{THz}$ bandwidth range.

Acknowledgement: This work was partially supported by the EPSRC via projects EP/J017582/1 and EP/L000091/1 (UNLOC and PEACE)

\section{Conclusions}

We propose and numerically validate, for the first time, a closed form approximation of FWM efficiency in lumped optical transmission systems that deploy mid-link OPC. The theory and numerical results show that lumped system deploying mid-ink OPC can significantly compensate FWM power resulted from strongly phase matched signalsignal interaction. Modulated optical signal transmission through lumped system with mid-link OPC can get significant performance improvements when either using small signal bandwidth with standard span length (of $80 \mathrm{~km})$, or high bandwidth with smaller span length $(10 \mathrm{~km})$.

\section{References}

[1] A. D. Ellis, "The nonlinear Shannon limit and the need for new fibres," Nonlinear Opt. Appl. VI 8434, 84340H-84340H-10 (2012).

[2] X. Chen and W. Shieh, "Closed-form expressions for nonlinear transmission performance of densely spaced coherent optical OFDM systems.," Opt. Express 18, 19039-19054 (2010).

[3] D. A. Cleland, A. D. Ellis, and C. H. F. Sturrock, "Precise modelling of four wave mixing products over $400 \mathrm{~km}$ of step-index fibre," Electron. Lett. 28, 1171 (1992).

[4] A. Yariv, D. Fekete, and D. M. Pepper, "Compensation for channel dispersion by nonlinear optical phase conjugation.," Opt. Lett. 4, 52 (1979).

[5] J. Ania-Castañón, "Quasi-lossless transmission using second-order Raman amplification and fibre Bragg gratings.," Opt. Express 12, 43724377 (2004).

[6] M. E. McCarthy, M. A. Z. Al Kahteeb, F. M. Ferreira, and A. D. Ellis, "PMD tolerant nonlinear compensation using in-line phase conjugation," Opt. Express 24, 3385-3392 (2016)

[7] D. Rafique and A. D. Ellis, "Impact of signal-ASE four-wave mixing on the effectiveness of digital back-propagation in $112 \mathrm{~Gb} / \mathrm{s}$ PM-QPSK systems.," Opt. Express 19, 3449-54 (2011).

[8] Mohammad A. Z. Al-Khateeb, Mary McCarthy, Christian Sánchez, and Andrew Ellis, "Effect of second order signal-noise interactions in nonlinearity compensated optical transmission systems," Opt. Lett. 41, 1849-1852 (2016).

[9] Pierluigi Poggiolini, "The GN Model of Non-Linear Propagation in Uncompensated Coherent Optical Systems," J. Lightwave Technol. 30, 3857-3879 (2012) 